\title{
Shallow-Trench-Isolation (STI)-Induced Mechanical-Stress-Related Kink-Effect Behaviors of 40-nm PD SOI NMOS Device
}

\author{
V. C. Su, James B. Kuo, Fellow, IEEE, I. S. Lin, Guan-Shyan Lin, David C. Chen, \\ Chune-Sin Yeh, Member, IEEE, Cheng-Tzung Tsai, and Mike Ma
}

\begin{abstract}
This brief reports the shallow-trench-isolation (STI)induced mechanical-stress-related kink-effect behaviors of the 40-nm PD silicon on insulator (SOI) NMOS device. As verified by the experimentally measured data and the 2-D simulation results, the kink-effect behaviors occur at a higher $V_{D}$ in the saturation region and show a less steep subthreshold slope for the 40-nm PD device with a smaller S/D length of $0.17 \mu \mathrm{m}$ due to the weaker function of the parasitic bipolar device as a result of the larger body-source bandgap-narrowing effect coming from the higher STI-induced mechanical stress.
\end{abstract}

Index Terms-CMOSFET, silicon on insulator (SOI) technology.

\section{INTRODUCTION}

$\mathbf{M}$ ECHANICAL stress induced by shallow trench isolation (STI) may affect the performance of CMOS devices [1], [2]. Mechanical stress may change work function, effective mass, carrier mobility, and junction leakage [3]. Silicon on insulator (SOI) has been becoming another mainstream technology for CMOS VLSI in the nanometer regime [4]. For nanometer PD SOI CMOS devices, the S/D region may be very small; the STI-induced mechanical stress is not negligible. The effects of mechanical stress on the performance of the SOI MOS devices with STI have been studied [2]. Until now, no papers about the STI-induced effects on the performance of the PD SOI CMOS devices could be found. In this brief, STI-induced mechanical-stress-related kink-effect behaviors of the 40-nm PD SOI NMOS device are reported. In the following sections, the STI-induced mechanical stress is described first, followed by STI-induced kink effects, discussion, and conclusion.

\section{STI-InduCED Mechanical StRess}

The 40-nm PD SOI NMOS test device fabricated in the industry with its cross section, as shown in Fig. 1(a) and (b), has a 70-nm thin film doped with a p-type doping density of

Manuscript received December 27, 2007. This work was supported by the Semiconductor Research Corporation under a Research Grant. The review of this brief was arranged by Editor H. S. Momose.

V. C. Su, J. B. Kuo, and I. S. Lin are with the Department of Electrical Engineering, National Taiwan University, Taipei 106-17, Taiwan, R.O.C. (e-mail: jbkuo@cc.ee.ntu.edu.tw).

G.-S. Lin, D. C. Chen, C.-S. Yeh, C.-T. Tsai, and M. Ma are with the ATD Modeling Division, United Microelectronics Corporation, Hsinchu 300, Taiwan, R.O.C

Color versions of one or more of the figures in this brief are available online at http://ieeexplore.iee. org.

Digital Object Identifier 10.1109/TED.2008.922858

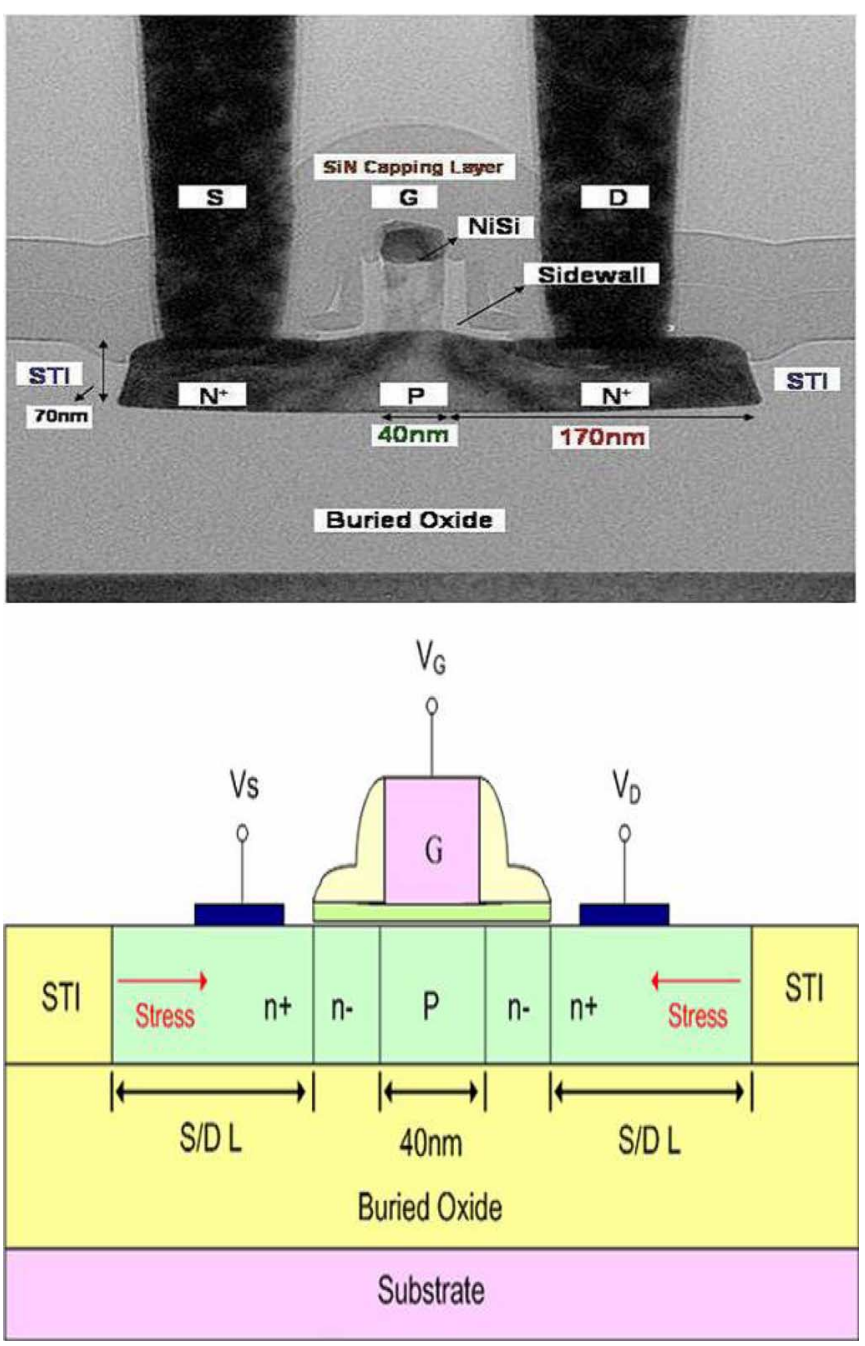

Fig. 1. (a) TEM cross section of the 40-nm PD SOI NMOS device under study. (b) Cross section of the PD SOI NMOS device used in the Medici simulation.

$3 \times 10^{18} \mathrm{~cm}^{-3}$ above a buried oxide of $145 \mathrm{~nm}$, under a gate oxide of $1.5 \mathrm{~nm}$. A $65-\mathrm{nm}$ lightly doped drain region doped with an n-type density of $10^{19} \mathrm{~cm}^{-3}$ under a sidewall spacer has been adopted. In addition, a nickel polycide is formed on the top of the gate, and a SiN capping layer is deposited over the device. STI structure has been adopted in forming this PD SOI NMOS device. Two cases with the S/D length of 1.7 and $0.17 \mu \mathrm{m}$ have been designed for this brief. Experimental measurement of the 


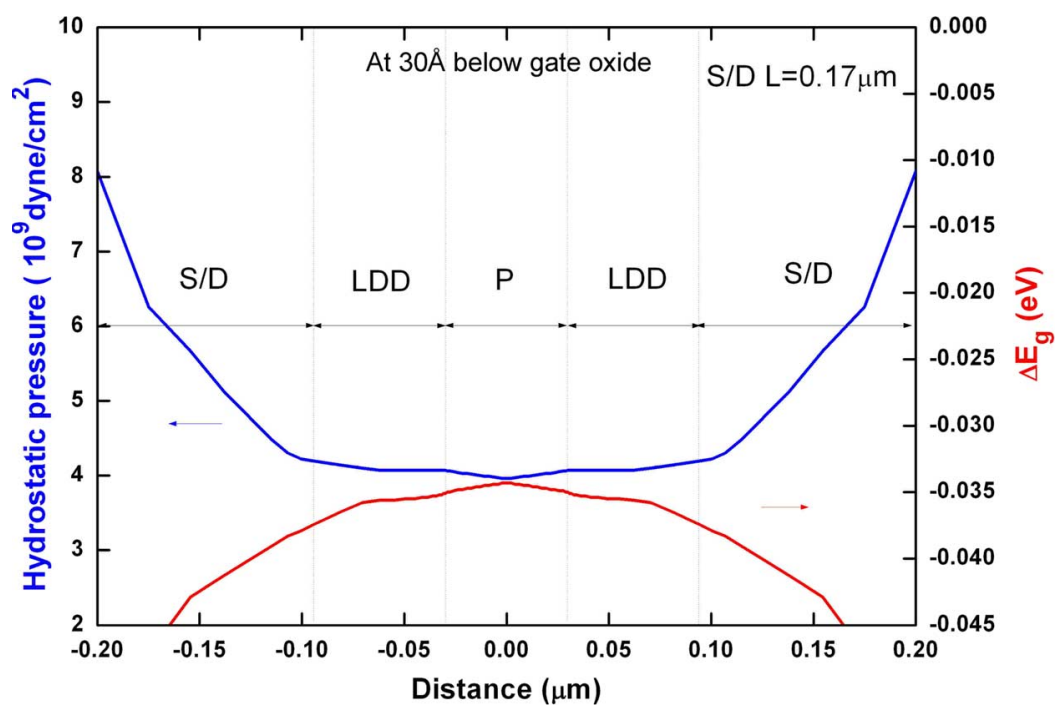

Fig. 2. Mechanical stress and bandgap-narrowing distribution in the lateral direction of the PD device with the S/D length of $0.17 \mu \mathrm{m}$.

test device and 2-D simulation considering mechanical-stress effects and energy-balance equation [5] have been used to carry out this brief. There are 8871 grids in the test device for the 2-D simulation.

Due to the trench isolation, the difference in the thermalexpansion coefficient between silicon and silicon dioxide, and the visco-elastic effects during the thermal anneals, a mechanical stress develops [2]. Using the result from a process simulator considering the hydrostatic pressure with the 2-D device simulator, Fig. 2 shows the hydrostatic pressure and the associated bandgap-narrowing distribution in the lateral direction of the 40-nm PD SOI NMOS device with S/D length of $0.17 \mu \mathrm{m}$ based on process-simulation results [6]. As induced by STI, the mechanical stress in terms of hydrostatic pressure is large in the S/D region near STI and decreases away from it. The corresponding bandgap narrowing, which is correlated to the mechanical stress, has a dramatic impact on the kink effect of the 40-nm PD NMOS device.

\section{STI-INDUCED KINK BEHAVIORS}

Fig. 3 shows $I_{\mathrm{D}}$ versus $V_{\mathrm{D}}$ of the 40-nm PD NMOS device with the S/D length of 1.7 and $0.17 \mu \mathrm{m}$, biased with its body floating, and based on the measured data and 2-D device simulation results using Medici. As shown in the figure, with a smaller S/D length of $0.17 \mu \mathrm{m}$, the kink-effect behavior occurs at a larger $V_{\mathrm{D}}$ as compared to the case with the S/D length of $1.7 \mu \mathrm{m}$. Fig. 4 shows the related output conductance versus $V_{\mathrm{D}}$ characteristics of the PD device biased at $V_{\mathrm{G}}=0.8 \mathrm{~V}$. With the shorter S/D length of $0.17 \mu \mathrm{m}$, the kink-effect behavior occurs at a larger $V_{\mathrm{D}}$ due to the higher bandgap-narrowing effect from the larger mechanical stress.

Fig. 5(a) and (b) shows the subthreshold characteristics of the 40-nm PD SOI NMOS device with the S/D length of 1.7 and $0.17 \mu \mathrm{m}$, biased at $V_{\mathrm{D}}=0.05 \mathrm{~V}$ and $0.8 \mathrm{~V}$ and body floating. At $V_{\mathrm{D}}=0.05 \mathrm{~V}$, the subthreshold curves are less steep than those at $V_{\mathrm{D}}=0.8 \mathrm{~V}$, since the parasitic bipolar device is not activated yet. At $V_{\mathrm{D}}=0.8 \mathrm{~V}$, the subthreshold slopes become steeper due to the turn-on of the bipolar device (the subthreshold-kink

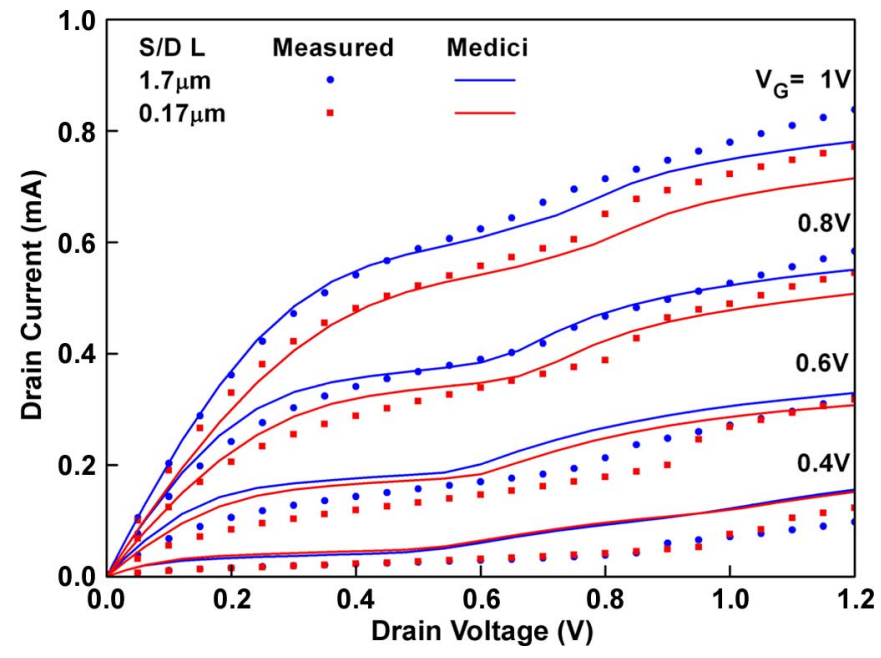

Fig. 3. $I_{\mathrm{D}}$ versus $V_{\mathrm{D}}$ of the 40-nm PD SOI NMOS device with the S/D length of 1.7 and $0.17 \mu \mathrm{m}$.

effect). With a smaller S/D length of $0.17 \mu \mathrm{m}$, the subthreshold slope becomes less steep as compared to the case with the S/D length of $1.7 \mu \mathrm{m}$, which implies a weaker action of the parasitic bipolar device due to the effect of more body-source bandgap narrowing from the STI-induced mechanical stress. The trends predicted by the 2-D device simulation are strongly correlated to the experimentally measured data, as shown in the figures.

\section{DISCUSSION}

The higher $V_{\mathrm{D}}$ at the onset of the kink-effect behavior in the saturation region and the less steepness of the subthresholdkink behavior of the 40-nm PD SOI NMOS device with the smaller S/D length of $0.17 \mu \mathrm{m}$ could be reasoned by considering the function of the parasitic bipolar device in the thin film. Fig. 6 shows the body-source voltage, which is the base-emitter voltage $\left(V_{\mathrm{BE}}\right)$ of the parasitic bipolar device in the thin film, versus $V_{\mathrm{D}}$ of the 40-nm PD NMOS device with the S/D length of $L=1.7$ and $0.17 \mu \mathrm{m}$, biased at $V_{\mathrm{G}}=0.4 \mathrm{~V}$ and $0.8 \mathrm{~V}$, based on the Medici simulation results. As shown in the figure, with 


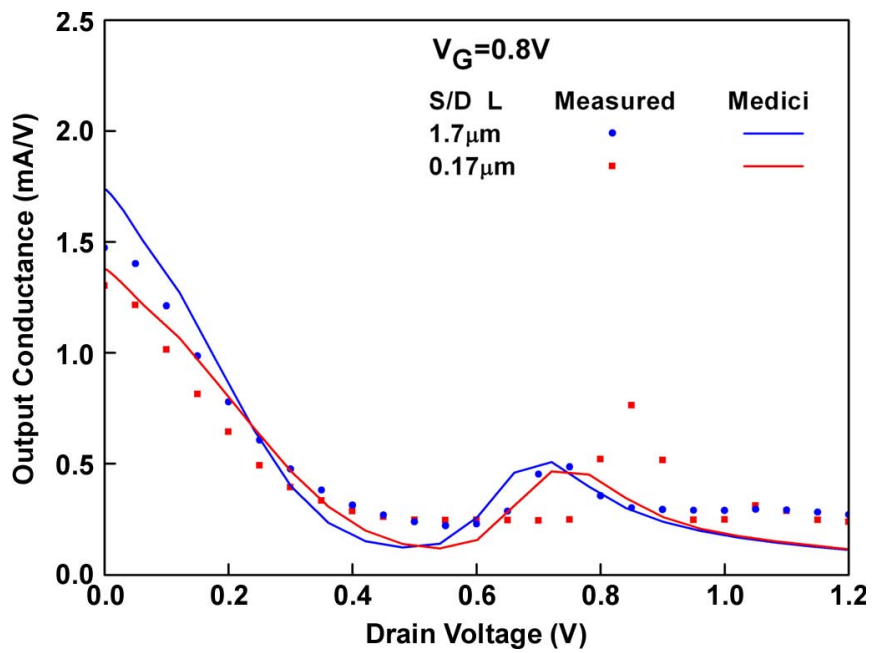

Fig. 4. Output conductance versus $V_{\mathrm{D}}$ of the 40-nm PD NMOS device with the S/D length of $L=1.7$ and $0.17 \mu \mathrm{m}$, biased at $V_{\mathrm{G}}=0.8 \mathrm{~V}$.

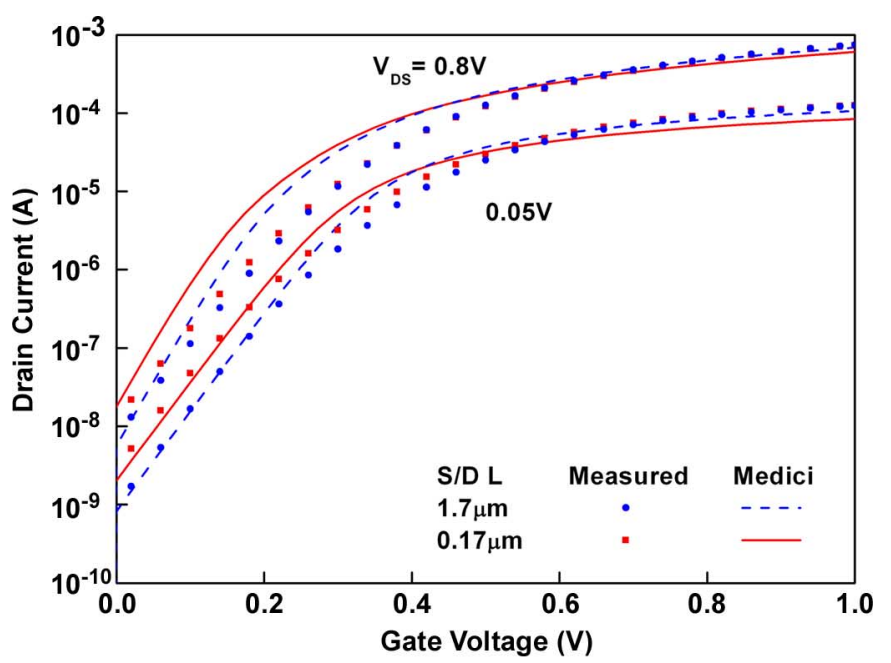

\begin{tabular}{|c|c|c|}
\hline S/D L & $0.05 \mathrm{~V}$ & $0.8 \mathrm{~V}$ \\
\hline $1.7 \mu \mathrm{m}$ & $79.9 \mathrm{mV} / \mathrm{dec}$ & $76.2 \mathrm{mV} / \mathrm{dec}$ \\
& & \\
\hline $0.17 \mu \mathrm{m}$ & $82.6 \mathrm{mV} / \mathrm{dec}$ & $81.4 \mathrm{mV} / \mathrm{dec}$ \\
\hline
\end{tabular}

Fig. 5. (a) Subthreshold characteristics of the 40-nm PD NMOS device biased at $V_{\mathrm{G}}=0.8$ and $0.05 \mathrm{~V}$. (b) Subthreshold $\mathrm{S}$ factor of the 40-nm PD NMOS device with the S/D length of $L=1.7$ and $0.17 \mu \mathrm{m}$, biased at $V_{\mathrm{G}}=0.8$ and $0.05 \mathrm{~V}$.

a smaller S/D length of $0.17 \mu \mathrm{m}$, the body-source voltage rises at a larger $V_{\mathrm{D}}$, which indicates the later turn-on the parasitic bipolar device. At the onset of the kink-effect behavior, the parasitic bipolar device is triggered to turn on with its current

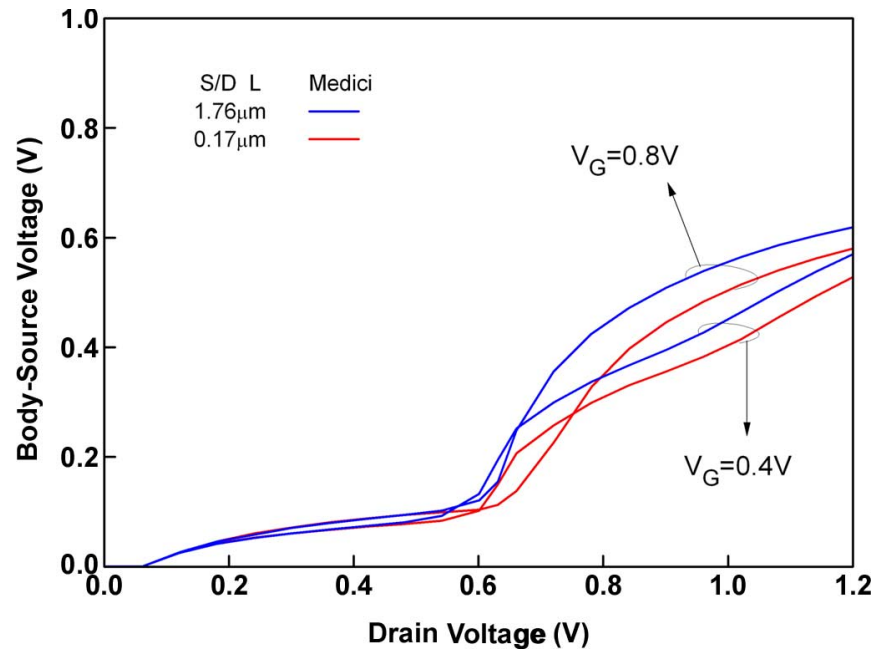

Fig. 6. Body-source voltage versus $V_{\mathrm{D}}$ of the 40-nm PD NMOS device with the S/D length of $L=1.7$ and $0.17 \mu \mathrm{m}$, biased at $V_{\mathrm{G}}=0.4$ and $0.8 \mathrm{~V}$, based on the Medici simulation results.

gain $\beta$ as a function of the difference in the bandgap narrowing between the base and the emitter [7]

$$
\beta \alpha \exp \left(\left(\Delta E_{\mathrm{gb}}-\Delta E_{\mathrm{ge}}\right) / k T\right)
$$

where $\Delta E_{\mathrm{gb}} / \Delta E_{\mathrm{ge}}$ is the bandgap narrowing in the base/the emitter (the source), $k$ is Boltzmann constant, and $T$ is temperature in kelvin. Due to the higher mechanical stress from STI, the bandgap narrowing in the emitter (source) $-\Delta E_{\text {ge }}$-is larger than that in the base (the channel) region $\Delta E_{\mathrm{gb}}$. Therefore, the function of the parasitic bipolar device in the thin film of the device with the shorter S/D length of $0.17 \mu \mathrm{m}$ becomes weaker as indicated by the smaller current gain. As a result, the onset of the kink-effect behavior occurs at a higher $V_{\mathrm{D}}$, and its subthreshold slope is less steep [8].

More implications could be obtained from studying the mechanical-stress effects on the device performance. Fig. 7(a) shows the drain voltage at the onset of the kink effect $-V_{\text {kink }}$-versus the S/D length of the PD SOI NMOS device biased at $V_{\mathrm{G}}=0.6$ and $0.8 \mathrm{~V}$, and Fig. 7(b) shows the $\mathrm{S}$ factor versus the S/D length of the PD SOI NMOS device biased at $V_{\mathrm{D}}=0.05$ and $0.8 \mathrm{~V}$, based on the 2-D simulation results. As shown in Fig. 7(a), until the S/D length is shrunk below $1.5 \mu \mathrm{m}$, the drain voltage $V_{\text {kink }}$ is insensitive to the S/D length. When the S/D length is smaller than $1.5 \mu \mathrm{m}, V_{\text {kink }}$ is sensitive to the S/D length. A smaller S/D length leads to a larger $V_{\text {kink }}$, which implies that the increased bandgapnarrowing effect slows down the turn-on of the parasitic bipolar device. As shown in Fig. 7(b), a similar trend could be seen on the S-factor plot. As the S/D length is smaller than $1.5 \mu \mathrm{m}$, the subthreshold slope of the device is sensitive to the S/D length. A smaller S/D length worsens the subthreshold slope due to the increased bandgap-narrowing effect, which weakens the function of the bipolar device. Therefore, the STI-induced mechanical-stress effect is important for the PD SOI NMOS devices with an S/D length smaller than $1.5 \mu \mathrm{m}$. The STIinduced mechanical-stress effects could be a key factor in determining the short-channel-effect behavior of the PD SOI CMOS device in the nanometer regime. 

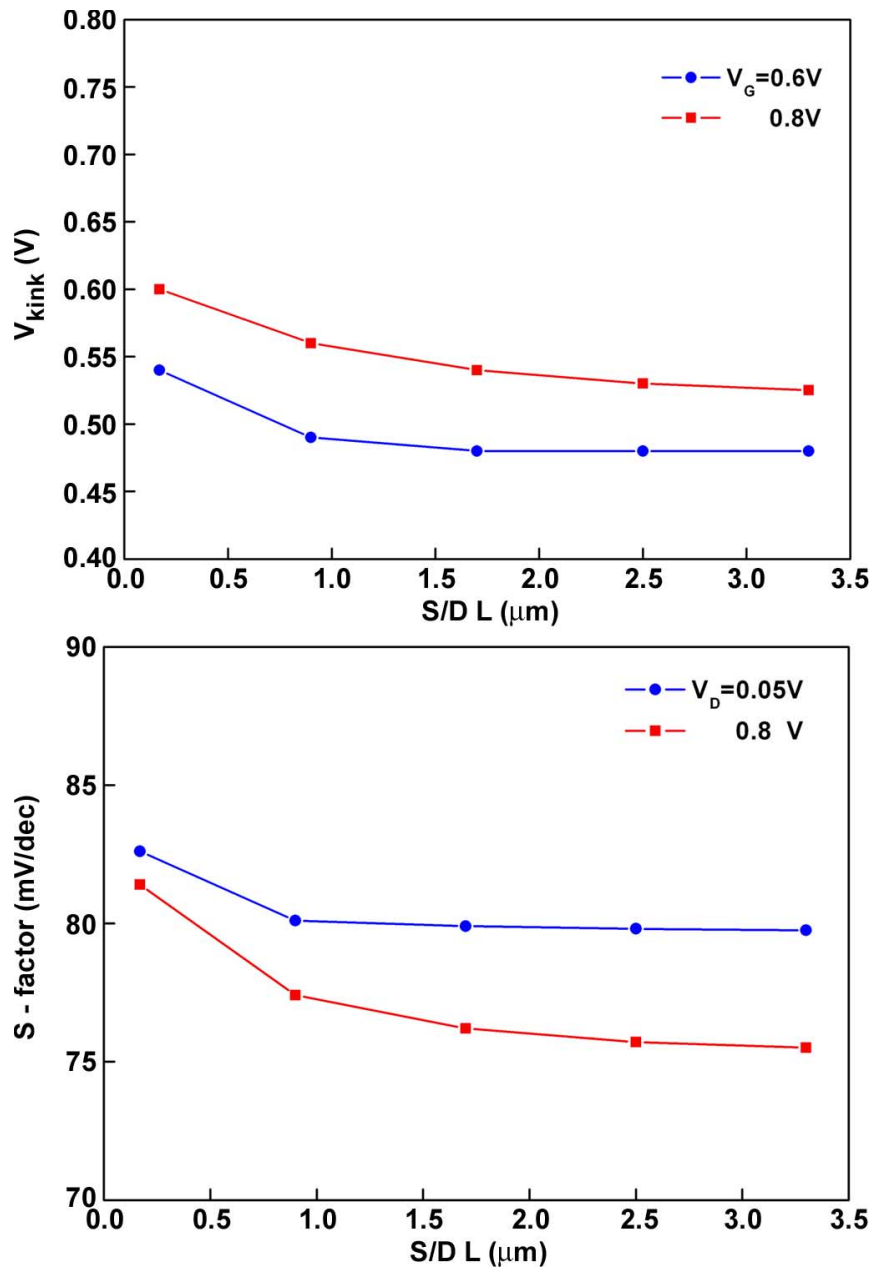

Fig. 7. (a) Drain voltage at the onset of the kink effect $-V_{\text {kink }}-$ versus the S/D length of the PD SOI NMOS device biased at $V_{\mathrm{G}}=0.6$ and $0.8 \mathrm{~V}$, and (b) $\mathrm{S}$ factor versus the S/D length of the PD SOI NMOS device biased at $V_{\mathrm{D}}=$ 0.05 and $0.8 \mathrm{~V}$, based on 2-D simulation results.

\section{CONCLUSION}

This brief reports the STI-induced mechanical-stress-related kink-effect behaviors of the 40-nm PD SOI NMOS device. As verified by the experimentally measured data and the 2-D simulation results, the kink-effect behaviors occur at a higher $V_{\mathrm{D}}$ in the saturation region and show a less steep subthreshold slope for the 40-nm PD device with a smaller S/D length of $0.17 \mu \mathrm{m}$ due to the higher body-source bandgap narrowing coming from the higher STI-induced mechanical stress.

\section{ACKNOWLEDGMENT}

The authors would like to thank S. C. Hsieh and R. Lee of UMC for their help.

\section{REFERENCES}

[1] R. A. Bianchi, G. Bouche, and O. Roux-dit-Buisson, "Accurate modeling of trench isolation induced mechanical stress effects on MOSFET electrical performance," in IEDM Tech. Dig., 2002, pp. 117-120.

[2] C. Gallon, G. Reimbold, G. Ghibaudo, R. A. Bianchi, R. Gwoziecki, S. Orain, E. Robilliart, C. Raynaud, and H. Dansas, "Electrical analysis of mechanical stress induced by STI in short MOSFETs using externally applied stress," IEEE Trans. Electron Devices, vol. 51, no. 8, pp. 12541260, Aug. 2004
[3] A. Hamada, T. Furusawa, N. Saito, and E. Takeda, "A new aspect of mechanical stress effects in scaled MOS devices," IEEE Trans. Electron Device, vol. 38, no. 4, pp. 895-900, Apr. 1991.

[4] J. B. Kuo and S. C. Lin, Low-Voltage SOI CMOS VLSI Devices and Circuits. New York: Wiley, 2001.

[5] Taurus Medici User Guide, Synopsys Inc., Mountain View, CA, Oct. 2005.

[6] Taurus TSUPREM-4 User Guide, Synopsys Inc., Mountain View, CA, Oct. 2005.

[7] T. C. Lu and J. B. Kuo, "An analytical bandgap-narrowing-related currentgain model for BJT devices operating at 77 K," Solid State Electron., vol. 35, no. 6, pp. 785-790, Jun. 1992.

[8] S. C. Lin and J. B. Kuo, "Temperature-dependent kink effect model for partially-depleted SOI NMOS devices," IEEE Trans. Electron Devices, vol. 46, no. 1, pp. 254-258, Jan. 1999.

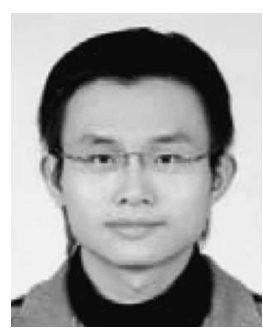

V. C. Su was born in Nantou, Taiwan, R.O.C., in 1983. He received the B.S. degree in electrical engineering from the Chin-Yi Institute of Technology, Taichung, Taiwan, in 2005 and the M.S. degree in electrical engineering from the National Taiwan University, Taipei, Taiwan, in 2008.

His research interest is the field of CMOS devices.

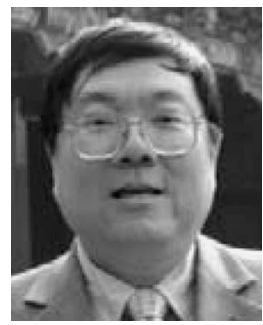

James B. Kuo (S'85-M'85-SM'92-F'00) received the $\mathrm{Ph} . \mathrm{D}$. degree in electrical engineering from Stanford University, Stanford, CA, in 1985.

From 1985 to1987, he was a Research Associate with the IC Laboratory of Stanford. Since 1987, he has been with the Department of Electrical Engineering, National Taiwan University (NTU), Taipei, Taiwan, R.O.C., where he is currently a Professor. Between 2000 and 2002, he was a Professor with the University of Waterloo, Waterloo, ON, Canada. His research expertise is in the field of low-voltage CMOS VLSI circuits and compact modeling of CMOS VLSI devices. He has published 300 technical papers and authored eight textbooks on CMOS VLSI. He has graduated 80 graduate students who specialized in CMOS VLSI and who are now working in leading microelectronics companies.

Dr. Kuo served as the Vice President of Membership for the IEEE Electron Devices Society. He was awarded the prestigious Canada Research Chair on Low-Voltage CMOS VLSI from the University of Waterloo. In 2007, he was awarded the prestigious Life Distinguished Professor at NTU.

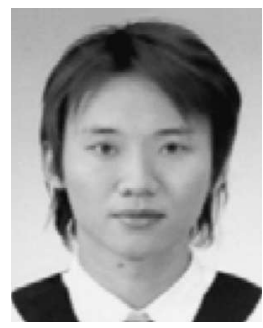

I. S. Lin was born in Kaohsiung, Taiwan, R.O.C., in 1983. He received the B.S. degree in electrical engineering from the National Sun Yat-Sen University, Kaohsiung, in 2005 and the M.S. degree in electrical engineering from the National Taiwan University, Taipei, Taiwan, in 2008.

His research interest is the field of CMOS devices.

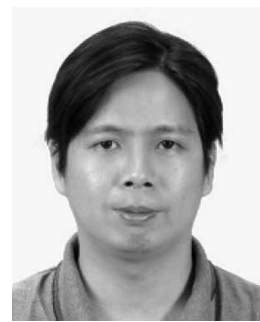

Guan-Shyan Lin was born in Taipei, Taiwan, R.O.C. He received the B.S. degree in electrical engineering from Tamkang University, Taipei, in 1996, and the M.S. degree in electrical engineering from the University of Florida, Gainesville, in 2002.

Since December 2002, he has been with the Advanced Technology Development Modeling Division, United Microelectronics Corporation, Hsinchu, Taiwan. His current research interest focuses on the characterization and modeling of advanced SOI and multigate devices. 


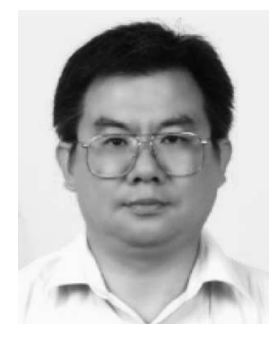

David C. Chen received the Ph.D. degree in electrical engineering from the University of Southern California, Los Angeles, in 1997.

He joined the United Microelectronics Corporation, Hsinchu, Taiwan, R.O.C., in 1997, where he is currently with the Advanced Technology Development Group. His current research interests include baseband and radio frequency (RF) device modeling of nanometer technology, RF reliability, RF and mixed-signal circuit design, and neural network application for communication system.

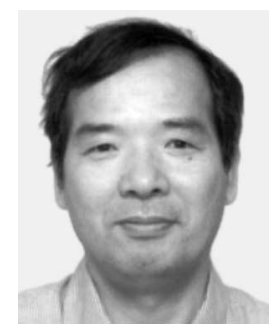

Chune-Sin Yeh (M'83) was born in Hsinchu, Taiwan, R.O.C. He received the B.S. degree in electrical engineering from the National Cheng-Kung University, Tainan, Taiwan, and the Ph.D. degree in electrical engineering from the University of Florida, Gainesville.

Since July 2005, he has been in charge of the Advanced Technology Development Modeling Division, United Microelectronics Corporation (UMC), Hsinchu. Before he joined UMC, he was with National Semiconductor, BTA Technology, Celestry, and Cadence, all in the area of technology CAD (TCAD) and TCAD-related tools and consulting service business operations.

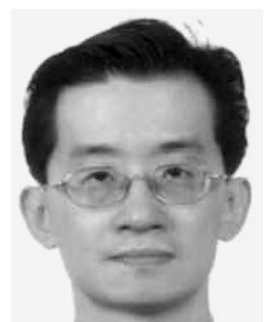

Cheng-Tzung Tsai received the Ph.D. degree in electrophysics from the National Chiao Tung University, Hsinchu, Taiwan, R.O.C.

Since 1998, he has been with the United Microelectronics Corporation (UMC), Hsinchu, Taiwan, R.O.C., where he is currently the Senior Manager of the Exploratory Technologies Division, Advanced Technology Development Group, responsible for the development of SOI, strain engineering, Ge-channel transistor, Schottky transistor, and emerging technologies beyond the 32-nm node. Prior to UMC, he was with the Mosel Corporation for DRAM FEoL process development and device characteristic analysis.

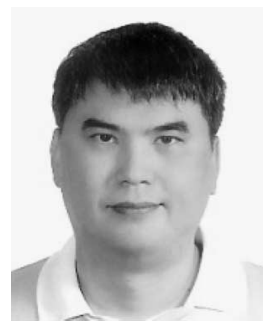

Mike Ma received the $\mathrm{Ph} . \mathrm{D}$. degree in materials science and engineering from North Carolina State University, Raleigh, in 1991.

$\mathrm{He}$ is currently the Director of the Exploratory Technologies Division, Advanced Technology Development Group, United Microelectronics Corporation (UMC), responsible for the assessment and development of emerging technologies beyond the 32-nm node. Prior to his current post, he held numerous management positions in engineering within UMC, dating back to 2000 . 